\title{
Life-History Variation Predicts the Effects of Demographic Stochasticity on Avian Population Dynamics
}

\author{
Bernt-Erik Sæther, ${ }^{1, *}$ Steinar Engen, ${ }^{2}$ Anders Pape Møller, ${ }^{3}$ Henri Weimerskirch, ${ }^{4}$ Marcel E. Visser ${ }^{5}$ \\ Wolfgang Fiedler, ${ }^{6}$ Erik Matthysen, ${ }^{7}$ Marcel M. Lambrechts, ${ }^{8}$ Alexander Badyaev, ${ }^{9}$ Peter H. Becker, ${ }^{10}$ \\ Jon E. Brommer, ${ }^{11}$ Dariusz Bukacinski, ${ }^{12}$ Monika Bukacinska, ${ }^{12}$ Hans Christensen, ${ }^{13}$ Janis Dickinson, ${ }^{14}$ \\ Chris du Feu, ${ }^{5}$ Frederick R. Gehlbach, ${ }^{15}$ Dik Heg, ${ }^{16, \dagger}$ Hermann Hötker, ${ }^{17}$ Juha Merilä, ${ }^{11}$ Jan Tøttrup Nielsen, ${ }^{18}$ \\ Wallace Rendell, ${ }^{19}$ Raleigh J. Robertson, ${ }^{20}$ David L. Thomson, ${ }^{5}$ János Török, ${ }^{21}$ and Piet Van Hecke ${ }^{22}$
}

1. Department of Biology, Norwegian University of Science and Technology, N-7491 Trondheim, Norway;

2. Department of Mathematical Sciences, Norwegian University of Science and Technology, N-7491 Trondheim, Norway;

3. Laboratoire de Parasitologie Evolutive, Centre National de la Recherche Scientifique (CNRS)-Unité Mixte de Recherche 7103, Université Pierre et Marie Curie, Bâtiment A, 7ème étage, 7 quai St. Bernard, Case 237, FR75252 Paris Cedex 05, France;

4. Centre de Etudes Biologiques de Chizé-CNRS, FR-79360 Villiers en Bois, France;

5. Netherlands Institute of Ecology, P.O. Box 40, NL-6666 ZG Heteren, The Netherlands;

6. Max-Planck-Forschungsstelle für Ornithologie, Andechs, und Radolfzell, Vogelwarte Radolfzell, Schloss Möggingen, D-78315 Radolfzell, Germany;

7. Laboratory of Animal Ecology, Department of Biology, University of Antwerp, B-2610 Antwerp, Belgium;

8. Centre d'Ecologie Fonctionnelle et Evolutive, CNRS, 1919 Route de Mende, F-34293 Montpellier Cedex 5, France;

9. Ecology and Evolutionary Biology, University of Arizona, Tucson, Arizona 85721;

10. Institut für Vogelforschung, Vogelwarte Helgoland, An der Vogelwarte 21, D-26386 Wilhelmshaven, Germany;

11. Department of Ecology and Systematics, P.O. Box 65 (Biocenter 3, Viikinkaari 1), FIN-00014 University of Helsinki, Finland;

12. Centre for Ecological Research, Polish Academy of Sciences, Dziekanow Lesny, ul. M. Konopnickiej 1, PL-05-092 Lomianki, Poland;

13. Tved 107C, DK-6270 Tønder, Denmark;

14. Museum of Vertebrate Zoology, Hastings Natural History Reservation, Carmel Valley, California 93924;

15. Department of Biology, Baylor University, Waco, Texas 76798-7388;

16. Zoological Laboratory, University of Groningen, P.O. Box 14, 9750 AA Haren, The Netherlands;

17. Naturschutzbund Deutschland Institut für Vogelschutz, Goosstroot 1, D-24861 Bergenhusen, Germany;

18. Espedal 4, DK-9870 Sindal, Denmark;

19. Applied Sciences and Computing, Loyalist College, Wallbridge-Loyalist Road, P.O. Box 4200, Belleville, Ontario K8N 5B9, Canada;

20. Department of Biology, Queen's University, Kingston, Ontario K7L

3N6, Canada;

21. Behavioural Ecology Group, Department of Systematics, Zoology, and

* Corresponding author; e-mail: bernt-erik.sather@bio.ntnu.no.

† Present address: Zoological Laboratory and Behavioural Ecology, Zoology Department, University of Bern, Wohlenstrasse 50a, CH-3032 Hinterkappelen, Switzerland.

Am. Nat. 2004. Vol. 164, pp. 793-802. () 2004 by The University of Chicago. 0003-0147/2004/16406-30240\$15.00. All rights reserved.
Ecology, Eötvös University, H-1117, Budapest, Pázmány P. sétány 1/C, Hungary;

22. Putsesteenweg 147, B-2920 Kalmthout, Belgium

Submitted June 23, 2003; Accepted May 25, 2004;

Electronically published November 9, 2004

Online enhancement: table.

ABSTRACT: Comparative analyses of avian population fluctuations have shown large interspecific differences in population variability that have been difficult to relate to variation in general ecological characteristics. Here we show that interspecific variation in demographic stochasticity, caused by random variation among individuals in their fitness contributions, can be predicted from a knowledge of the species' position along a "slow-fast" gradient of life-history variation, ranging from high reproductive species with short life expectancy at one end to species that often produce a single offspring but survive well at the other end of the continuum. The demographic stochasticity decreased with adult survival rate, age at maturity, and generation time or the position of the species toward the slow end of the slow-fast life-history gradient. This relationship between life-history characteristics and demographic stochasticity was related to interspecific differences in the variation among females in recruitment as well as to differences in the individual variation in survival. Because reproductive decisions in birds are often subject to strong natural selection, our results provide strong evidence for adaptive modifications of reproductive investment through life-history evolution of the influence of stochastic variation on avian population dynamics.

Keywords: demographic stochasticity, environmental stochasticity, life-history variation, stochastic population dynamics, birds.

Stochastic factors strongly affect the population dynamics of many bird populations (Lebreton 1990; Pimm 1991; Stacey and Taper 1992; Newton 1998; Bro et al. 2000; Lande et al. 2003). To answer one of the most central ecological questions of why there are such large interspe- 
cific differences in population variability (Pimm 1991; Ariño and Pimm 1995; Inchausti and Halley 2002), we must therefore obtain a proper understanding of how the separate effects of demographic and environmental stochasticity are related to demographic variation across species. Theoretical analyses have shown that knowledge of demographic stochasticity is especially important for fluctuations of small populations (May 1973; Leigh 1981; Lande 1998; Lande et al. 2003) that often are of management concern. For instance, important characteristics such as the expected time to extinction (Lande 1998; Sæther and Engen 2003) and the presence of stochastic Allee effects at small population sizes (Lande 1998; Engen et al. 2003) are closely related to the magnitude of the demographic variance $\sigma_{\mathrm{d}}^{2}$. As a consequence, failure to include demographic stochasticity in the analyses may result in severely biased predictions of population viability (Sæther and Engen 2002a, 2003). However, estimates of $\sigma_{\mathrm{d}}^{2}$ are available only for a few species (Lande et al. 2003).

Here we relate interspecific variation in $\sigma_{\mathrm{d}}^{2}$ to avian lifehistory characteristics that are easier to collect than longterm data on individual fitness variation that are necessary for estimating $\sigma_{\mathrm{d}}^{2}$ (Lande et al. 2003). Such information can therefore be used to get approximate information about $\sigma_{\mathrm{d}}^{2}$ in many rare or endangered species. Furthermore, recent comparative studies have suggested that in many taxa, interspecific variation in demographic variables can be predicted from knowledge of life-history characteristics because demographic traits such as adult survival or age at maturity decreases with clutch size (Pfister 1998; Sæther and Bakke 2000; Gaillard and Yoccoz 2003). Furthermore, in birds and mammals not only mean values but also information about variability in demographic traits can be gained from knowledge of life-history characteristics. For instance, in birds (Sæther and Bakke 2000) and mammals (Gaillard and Yoccoz 2003), the relative contribution of temporal variation in different demographic traits to fluctuations in the population growth rates is dependent on the life-history characteristics of the species. Such relationships can be due to life-history-dependent effects of either density dependence (e.g., Lande et al. 2002) or stochastic variables. Here we examine how the total stochastic contribution to annual variation in population size depends on the life-history characteristics of the species. We do this by relating $\sigma_{\mathrm{d}}^{2}$ to environmental stochasticity $\sigma_{\mathrm{e}}^{2}$, random variation affecting the whole population or group of individuals similarly (Lande et al. 2003), obtained from analysis of time series. The presence of such a relationship will indicate a link between environmental fluctuations, life-history variation, and patterns in avian population dynamics.

Previous analyses have shown that birds can be divided along a "slow-fast" continuum of life-history variation
(Sæther and Bakke 2000; Bennett and Owens 2002). At one end of the continuum, we have species that are characterized by an early age at maturity, large clutch sizes, and high mortality rates. At the opposite end are longlived species that produce a few (often a single) offspring and have a delayed onset of reproduction. Two alternative hypotheses may explain covariation between $\sigma_{\mathrm{d}}^{2}$ and the position of a species along this slow-fast continuum. According to one hypothesis, $\sigma_{\mathrm{d}}^{2}$ is expected to increase with adult survival rate (and hence to decrease with clutch size) because very few offspring recruit in short-lived species with a high first-year mortality. Alternatively, $\sigma_{\mathrm{d}}^{2}$ can be expected to decrease with adult survival rate because lifehistory constraints (small reproductive rates, high life expectancy) generate small variability in fitness among individuals in long-lived species. Here we examine the validity of these hypotheses using a data set of individual variation in fitness contribution of 31 bird species.

\section{Methods \\ Demographic Variance}

The demographic variance $\sigma_{\mathrm{d}}^{2}$ was estimated from data on individual variation among females in their fitness contributions to the following generations (for further discussion of the rationale behind this definition, see Engen et al. 1998; Lande et al. 2003). The total contribution of a female $i$ in year $t\left(R_{i}\right)$ is the number of female offspring born during the year that survive for at least 1 year plus one more if the female survives to the next year (Sæther et al. 1998). The demographic variance was estimated (Lande et al. 2003) as the weighted mean across years of $\sigma_{\mathrm{d}}^{2}(t)=\mathrm{E}[1 /(a-1)] \sum\left(R_{i}-\bar{R}\right)^{2}$, where $\bar{R}$ is the mean contribution of the females, $a$ is the number of recorded contributions in year $t$, and $\mathrm{E}$ denotes the expectation.

The demographic variance can be partitioned (Fox and Kendall 2002) into components due to variation in fecundity, survival, and interaction between fecundity and survival. Writing $B$ for the number of offspring produced, $I=1$ if the mother survives and $I=0$ if she dies, the demographic variance is the mean over years of the withinyear variance of $R=B+I$. This can be split into its components $\operatorname{Var}(R)=\operatorname{Var}(B)+\operatorname{Var}(I)+2 \operatorname{Cov}(B, I)$, which can be estimated separately by simple sum of squares.

In long-lived species, stochastic variation in age structure constitutes an important component of demographic stochasticity (Goodman 1967). For species with a mean age of maturity older than 3 years, we estimated $\sigma_{\mathrm{d}}^{2}$ by the method of S. Engen, R. Lande, and B.-E. Sæther (unpublished manuscript). Based on the contributions $\left(B_{j p} I_{j t}\right)$ for the different age classes $i$ in year $t$, we calculate the demographic stochasticity from the projection matrix (Cas- 
well 2001) and separate this into components that are generated by demographic stochasticity in each vital rate. Let $a_{t}$ denote the individual observations of the number of female offspring and mother's survival in year $t$ for age class $i$, with $j=1,2, \ldots, a_{t}$. Then the unbiased estimators for the component of $\sigma_{\mathrm{d}}^{2}$ from fecundity $F_{i}$, survival $S_{i}$, and the covariance between $F_{i}$ and $S_{i}\left(\operatorname{Cov}\left[F_{i}, S_{i}\right]\right)$ in age class $i$ are

$$
\begin{aligned}
\hat{\sigma}_{F_{i}}^{2} & =(C-T)^{-1} \sum_{t=1}^{T} \sum_{j=1}^{c_{t}}\left(B_{j t}-\bar{B}_{t}\right)^{2}, \\
\hat{\sigma}_{S_{i}}^{2} & =(C-T)^{-1} \sum_{t=1}^{T} \sum_{j=1}^{c_{t}}\left(I_{j t}-\bar{I}_{t}\right)^{2}, \\
\operatorname{Cov}\left(F_{i}, S_{i}\right) & =(C-T)^{-1} \sum_{t=1}^{T} \sum_{j=1}^{c_{t}}\left(B_{j t}-\bar{B}_{t}\right)\left(I_{j t}-\bar{I}_{t}\right),
\end{aligned}
$$

where $C=\sum_{t=1}^{T} a_{t}$ is the total number of individuals in age class $i$ and $T$ is the number of years. These estimates were computed separately for each age class to give an estimator for $\sigma_{\mathrm{d}}^{2}$ that is derived from the estimated deterministic Leslie matrix:

$$
\hat{\sigma}_{d}^{2}=\sum_{i=0}^{k} \frac{1}{U_{i}}\left[\left(\frac{\partial r}{\partial F_{i}}\right)^{2} \hat{\sigma}_{F_{i}}^{2}+\left(\frac{\partial r}{\partial S_{i}}\right)^{2} \hat{\sigma}_{S_{i}}^{2}+2 \frac{\partial r}{\partial F_{i}} \frac{\partial r}{\partial S_{i}} \hat{\operatorname{Cov}}\left(F_{i}, S_{i}\right)\right]
$$

Here $r=\ln \lambda$, where $\lambda$ is the dominant eigenvalue of the mean Leslie matrix and $\left(U_{0}, U_{1}, \ldots, U_{k}\right)$ is the stable age distribution for the deterministic matrix model.

In total, we obtained estimates of $\sigma_{\mathrm{d}}^{2}$ for 52 populations covering 31 species. Following the taxonomy of Sibley and Ahlquist (1990), we included 15 species from the order Passeriformes, 11 species from Ciconiiformes, three species from Strigiformes, and one species from each of the orders Piciformes and Gruiformes. For species composition, see table A1 in the online edition of the American Naturalist.

\section{Environmental Variance}

To examine the relationship between demographic and environmental stochasticity, we used long-term $(\geq 10$ years) time series of population fluctuations. We included only time series where the estimates were based on observations of recognizable individuals or direct nest counts to reduce the effects of sampling errors in the population estimates.

Following the approach of Sæther and Engen (2002b), two different models were used to analyze the time series. In the time series in which a visual inspection of the time series suggested an influence of density dependence (see appendix), we assumed a theta-logistic model of density regulation (Gilpin and Ayala 1973):

$$
\mathrm{E}(\Delta N \mid N)=r_{0} N\left[1-(N / K)^{\theta}\right]
$$

Here $r_{0}$ is the mean specific population growth rate as $N$ approaches $0, \Delta N$ is the change in population size $N$ from one year to another, $K$ is the carrying capacity, and $\theta$ specifies the form of the density regulation. By varying $\theta$, we can describe a wide variety of functions of density regulation. Equation (1a) can also be written (Sæther et al. 2000a)

$$
\mathrm{E}(\Delta N \mid N)=r_{1} N\left(1-\frac{N^{\theta}-1}{K^{\theta}-1}\right)
$$

where $r_{1}$ is the mean specific population growth rate at $N=1$. Writing $X=\ln N$ and using the first-order approximation $\Delta X \approx \Delta N / N$, we find for small to moderate fluctuations in population size that the expected change in log population size is

$$
\mathrm{E}(\Delta X \mid X)=s-\frac{r_{1}}{K^{\theta}-1}\left(e^{\theta X}-1\right)-\frac{1}{2} \sigma_{d}^{2} e^{-X}
$$

where $s=r_{1}-1 / 2 \sigma_{\mathrm{e}}^{2}$. Assuming $\sigma_{\mathrm{d}}^{2}$ to be known, the other parameters (including $\sigma_{\mathrm{e}}^{2}$ ) in equation (2) were estimated by maximum likelihood using the full likelihood function for the process obtained by assuming that $\Delta X$ conditioned on $X$ is normally distributed (see Sæther et al. 2000a, $2002 b, 2002 c$ ). However, in one species (Larus canus), no satisfactory fit to the theta-logistic model was obtained, and the estimate of $\sigma_{\mathrm{e}}^{2}$ was excluded from the analyses.

For steadily increasing or decreasing populations (see appendix) assumed to be far below $K$, we fitted a model with density-independent population growth (including demographic as well as environmental stochasticity) following the estimation procedures of Dennis et al. (1991) and Engen et al. (2001).

\section{Life-History Traits}

Modal values of clutch size and age at maturity $A$ were used as estimates of life-history variables. The mean recapture rate of individually recognizable breeding females was used as an estimate of adult survival rate $p$.

Assuming a stable population, we calculated the generation time as $T=A+p /(1-p)$ (Lande et al. 2003). For Diomedea exulans and Fulmarus glacoides, $P$ was set equal to .98 to avoid large effects on $T$ of small uncertainties in p. 


\section{Phylogenetic Analysis}

We used a generalized least squares approach to control for phylogeny and to estimate correlations between traits. The technique employed was suggested by Pagel (1999) and is outlined in detail by Freckleton et al. (2002). The approach fits a multivariate normal distribution to data using the phylogeny and an underlying assumption of Brownian trait evolution to specify the expected variance and covariance of traits among species in terms as a function of the phylogeny. The probability density for this model is

$$
p(\mathbf{y})=\frac{1}{(2 \pi|\Sigma \otimes \mathbf{V}|)^{n / 2}} \exp \left[-\frac{1}{2}(\mathbf{y}-\gamma \Gamma)^{\mathrm{T}}(\mathbf{\Sigma} \otimes \mathbf{V})^{-1}(\mathbf{y}-\gamma \Gamma)\right],
$$

where $\mathbf{y}$ is a $1 \times k n$ vector listing each of the $k$ traits for the $n$ species, $\Gamma$ is the design matrix, $\gamma$ is a $k \times 1$ list of the means of the $k$ traits, and $\Sigma$ is the $k \times k$ variancecovariance matrix of the traits. The matrix $\mathbf{V}$ is the variance-covariance matrix for the species, derived from the phylogeny. The elements of this matrix are shared branch lengths for species, thus measuring phylogenetic relatedness. Pagel (1999) defined an index $\tau$ that measures the degree of phylogenetic dependence. Index $\tau$ transforms the variance-covariance matrix $\mathbf{V}$ by multiplying the offdiagonal elements. A value of $\tau=0$ indicates that traits show complete phylogenetic independence, whereas a value of $\tau=1$ indicates that traits vary in accordance with the Brownian model.

Equation (3) may be used to generate a likelihood function that solves to yield an estimate of $\gamma$ for a given value of $\tau$ :

$$
\gamma^{*}=\left[\Gamma^{T} \mathbf{V}(\tau)^{-1} \mathbf{Y}\right]\left[\Gamma^{T} \mathbf{V}(\tau) \Gamma\right]^{-1}
$$

The elements of the variance-covariance matrix are then estimated by

$$
\Sigma^{*}=\frac{1}{(n-k)}(\mathbf{y}-\alpha \Gamma)^{T} \mathbf{V}(\tau)^{-1}(\mathbf{y}-\alpha \Gamma),
$$

where the divisor $(n-k)$ yields an unbiased (restricted) maximum likelihood estimate of the variance. The elements of $\Sigma$, the trait variance-covariance matrix, may be used to estimate the correlation between traits. Then $\tau$ is varied to find the value that maximizes the likelihood for the whole data set through a direct search algorithm.

In the results below, we report the maximum likelihood values of trait correlations (derived from $\Sigma$ ) as well as the maximum likelihood estimates of $\tau$ used to estimate these.

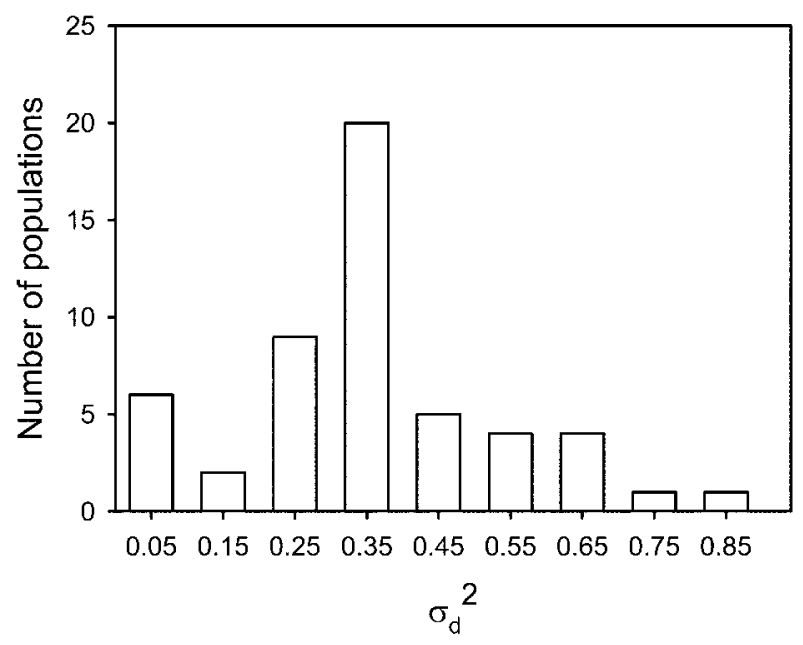

Figure 1: Distribution of estimates of demographic variance $\sigma_{\mathrm{d}}^{2}$ in 52 populations of 31 bird species.

\section{Results}

The estimates of $\sigma_{\mathrm{d}}^{2}$ ranged from 0.007 in one population of wandering albatross Diomedea exulans to 0.854 in the population of blue tit Parus caeruleus breeding on the island of Vlieland in the Netherlands (fig. 1). The mean value of $\sigma_{\mathrm{d}}^{2}$ across populations was $0.358(\mathrm{SD}=0.182)$. This shows that the demographic variance in bird populations is $<1$, with a majority of estimates in the interval from $\hat{\sigma}_{\mathrm{d}}^{2}=0.20$ to 0.40 .

We then decomposed the demographic stochasticity into different components due to random individual variation in fecundity and in survival. Using mean species-specific values, the two components of the demographic variance were positively correlated (fig. $2 a$, correlation coefficient $=0.39, n=30, P<.033$; after removing the effects of phylogeny, correlation coefficient $=0.28, n=30$, $P>.1[\tau=0.34]$; removing the outlier represented by Larus canus in the lower right-hand quadrant improved the correlation coefficient to $0.61, n=29, P<.001$ and $0.41, n=29, P<.05[\tau=0.84]$ before and after removing the effects of phylogeny, respectively). As expected from this relationship, interspecific differences in demographic variance were closely related to the size of both the fecundity component (fig. 2b, correlation coefficient $=0.76, n=30, P<.001$; phylogenetic correlation coefficient $=0.72, n=30, P<.001[\tau=0.37])$ and the survival component (fig. $2 c$, correlation coefficient $=0.86, n=30, P<.001$; phylogenetic correlation coefficient $=0.85, n=30, P<.001[\tau=0.35]$ ).

Interspecific differences in demographic stochasticity were well explained by life-history variation. Larger values of $\sigma_{\mathrm{d}}^{2}$ were found in species at the fast end of the avian 

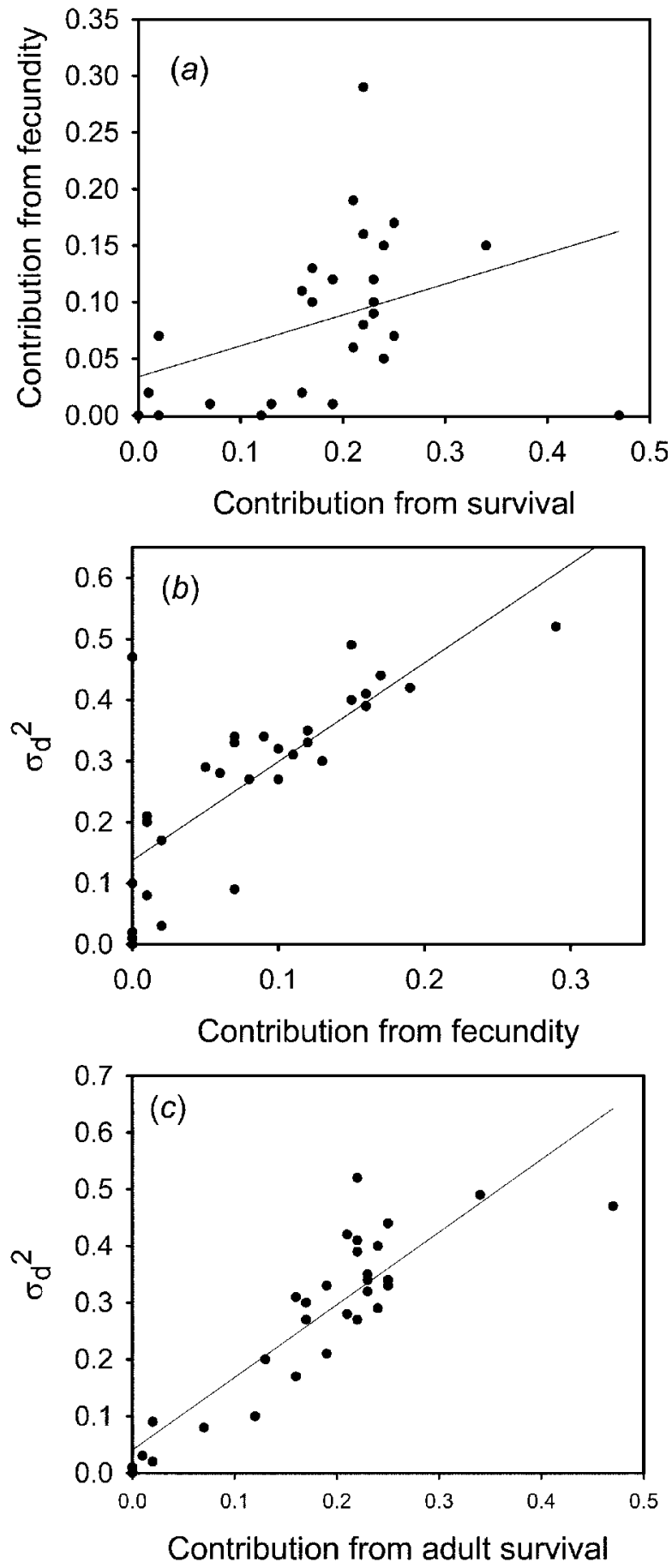

Figure 2: Relationship between the contribution of fecundity and survival to the demographic variance $\sigma_{\mathrm{d}}^{2}$ of 30 bird species $(a)$, and $\sigma_{\mathrm{d}}^{2}$ in relation to the contribution from fecundity $(b)$ and survival $(c)$. life-history continuum (Sæther and Bakke 2000; Bennett and Owens 2002), that is, in species with large clutch sizes (fig. $3 a$, correlation coefficient $=0.50, n=31, P=$ .004 ), short life expectancy (fig. $3 b$, correlation coefficient $=-0.57, n=31, P=.001$ ), early age at maturity (fig. $3 c$, correlation coefficient $=-0.61, n=31, P<$ .001 ) and short generation times (fig. $3 d$, correlation coefficient $=-0.56, n=31, P=.001$ ). However, a large phylogenetic component was present in those relationships (phylogenetic correlation coefficient $=0.18, P>$ $.05[\tau=1.10]$; phylogenetic correlation coefficient $=$ $-0.27, \quad P>.05 \quad[\tau=0.82]$; phylogenetic correlation coefficient $=-0.45, P<.05[\tau=0.94]$; and phylogenetic correlation coefficient $=-0.41, P<.05[\tau=1.11]$ for clutch size, adult survival rate, age at maturity, and generation time, respectively). The relationship between $\sigma_{\mathrm{d}}^{2}$ and $p$ was best explained by a curvilinear relationship (fig. $3 b$, phylogenetic correlation coefficient $=-0.71$, $P<.001[\tau=0.68])$, which may be explained by a reduction in the binomial variance component of $\sigma_{\mathrm{d}}^{2}$ from survival for $|p|<0.5$. The contribution of fecundity to the demographic variance increased with clutch size (correlation coefficient $=0.65, P<.001, n=30$; phylogenetic correlation coefficient $=-0.34, P<.05[\tau=1.29])$ but decreased with adult survival rate (correlation coefficient $=-0.51, P=.004, n=30$ ), age at maturity (correlation coefficient $=-0.55, P=.001, n=30$ ), and generation time (correlation coefficient $=-0.50, n=$ $30, P=.005)$. However, these latter three relations were strongly influenced by the phylogenetic relationships (phylogenetic correlations were $-0.11[\tau=1.12],-0.26$ $[\tau=1.07]$, and -0.17 [ $\tau=1.26], P>.05$ for adult survival rate, age at maturity, and generation time, respectively). Similarly, the contribution of adult survival decreased with adult survival rate (correlation coefficient $=-0.59, P<.001, n=30$; phylogenetic correlation coefficient $=-0.47, P<.01[\tau=0.72])$; age at maturity (correlation coefficient $=-0.59, \quad P<.001$, $n=30$; phylogenetic correlation coefficient $=-0.53$, $P<.01 \quad[\tau=0.73]$ ); and generation time (correlation coefficient $=-0.71, P<.001, n=30$; phylogenetic correlation coefficient $=-0.51, P<.01[\tau=0.82])$ but increased with clutch size (correlation coefficient $=0.45$, $P=.013, n=30$; not significant after accounting for phylogeny: phylogenetic correlation coefficient $=0.27$, $P>.05[\tau=0.93])$. This supports the hypothesis that the level of demographic stochasticity in avian population dynamics is subject to life-history constraints on the possible range of variation in fecundity or survival, resulting in small values of $\sigma_{\mathrm{d}}^{2}$ in long-lived species with small reproductive rates.

Environmental stochasticity is another major stochastic component influencing avian population dynamics (Le- 

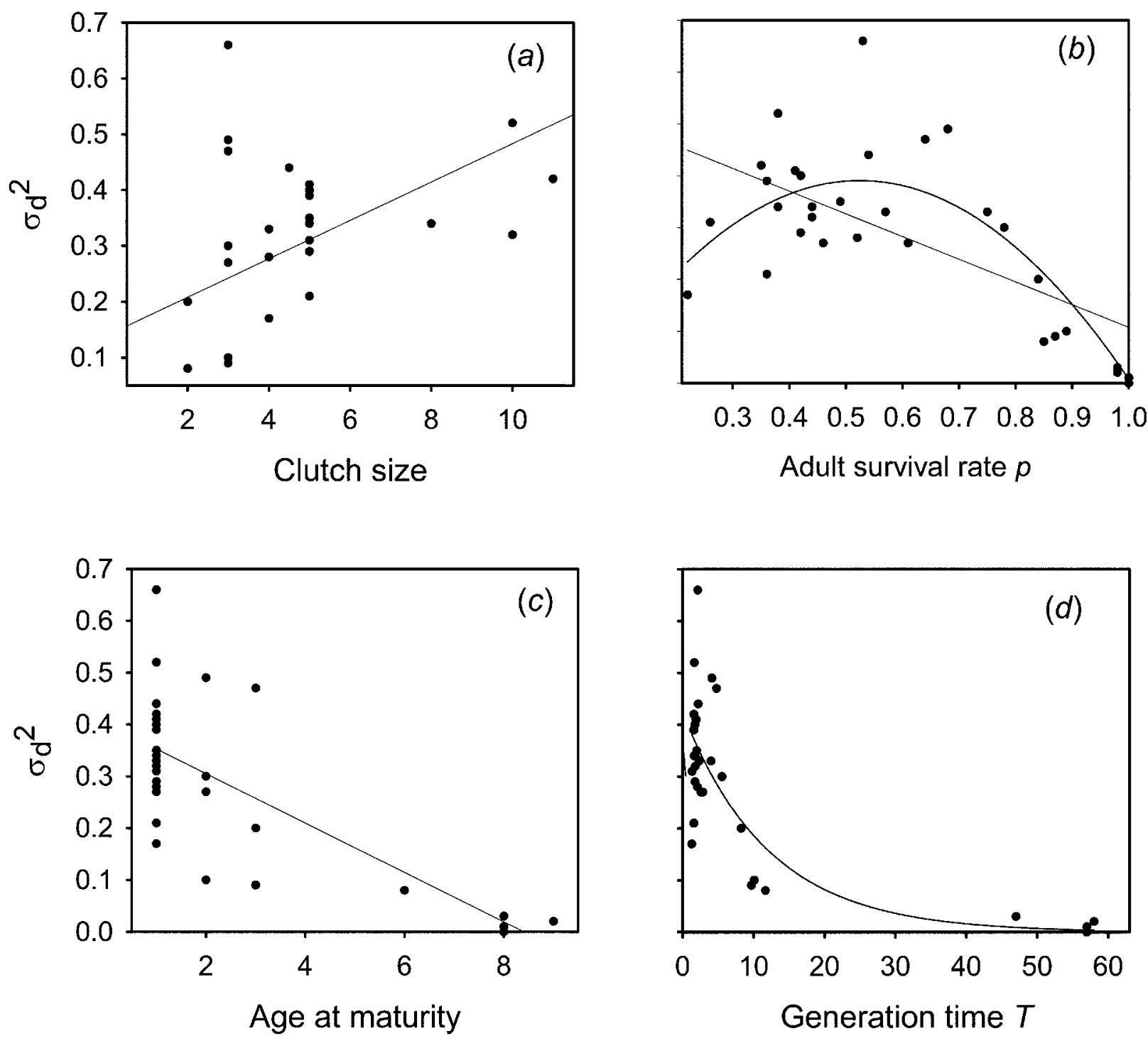

Figure 3: Mean value across species of the demographic variance $\sigma_{\mathrm{d}}^{2}$ in relation to clutch size $(a)$, adult survival rate $(b)$, age at maturity $(c)$, and generation time $(d)$. The equations for the lines of best fit was $\sigma_{\mathrm{d}}^{2}=-1.74 p^{2}+1.84 p-0.09$ and $\sigma_{\mathrm{d}}^{2}=-0.42 e^{-0.08 T}$ in $(b)$ and $(d)$, respectively.

breton 1990; Sæther et al. 2002a). The environmental variance $\sigma_{\mathrm{e}}^{2}$ can be estimated from time series analyses of long-term population fluctuations (see "Methods"). Again using the mean value across populations within species, we find that $\log _{10} \hat{\sigma}_{\mathrm{e}}^{2}$ and $\hat{\sigma}_{\mathrm{d}}^{2}$ were positively related (fig. 4; correlation coefficient $=0.51, P=.009, n=25)$. However, this relationship was no longer significant $(P>.05)$ after excluding Melopsiza melodia from the analysis or when accounting for the effects of phylogeny (phylogenetic correlation coefficient $=0.25, \tau=0.86$ ).

\section{Discussion}

This study is based on three fundamental assumptions. First, we assume that the large uncertainties in the parameter estimates (Sæther et al. 2000a; Sæther and Engen $2002 b$; Lande et al. 2003), because of time series that in a statistical sense are short, are independent of the speciesspecific life-history characteristics. To reduce the impact of violation of this assumption, we fit density-dependent models only to species with age at maturity $\leq 3$ years of 


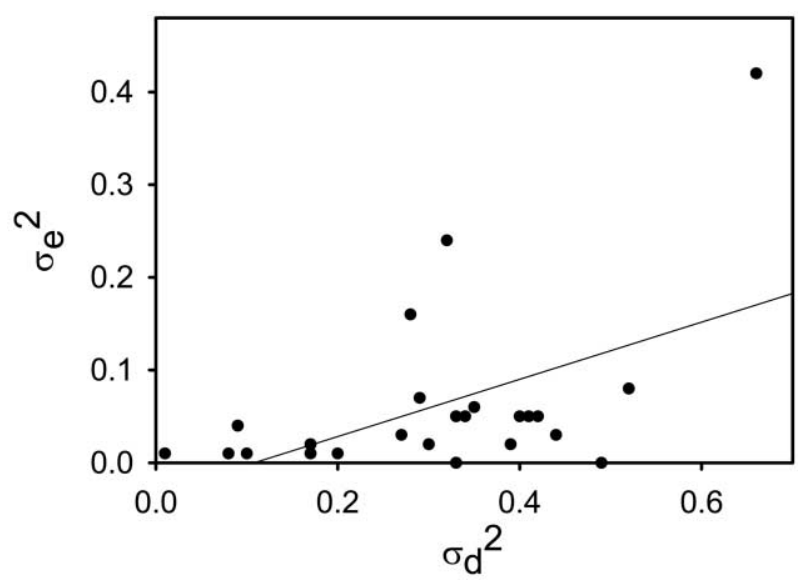

Figure 4: Mean value across species of environmental variance $\sigma_{\mathrm{e}}^{2}$ in relation to the demographic variance $\sigma_{\mathrm{d}}^{2}$ for species with age at maturity $<4$ years of age.

age (see appendix). Second, we assume that interspecific variation in population dynamical characteristics is larger than differences among populations within a species. This is not necessarily always the case. For instance, in three hole-nesting species (Parus caeruleus, Parus major, and Ficedula hypoleuca) for which several estimates of $\sigma_{\mathrm{d}}^{2}$ and $\sigma_{\mathrm{e}}^{2}$ were available (Sæther et al. 2003), the amongpopulation variation was larger than the interspecific variation in both the demographic $\sigma_{\mathrm{d}}^{2}$ and the environmental variance $\sigma_{\mathrm{e}}^{2}$. However, most of the estimates of long-lived species (appendix) still lie outside the range of intraspecific variation within these three passerine species that have quite similar life-history characteristics. Third, the estimates of $\sigma_{\mathrm{d}}^{2}$ may be influenced by interspecific differences in natal dispersal (Lambrechts et al. 1999) because small values of $\hat{\sigma}_{\mathrm{d}}^{2}$ are expected in populations where few recruits return. However, in several of the long-lived species with small demographic stochasticity (e.g., Diomedea exulans), a large proportion of all individuals in the population have been individually followed over several decades (Weimerskirch 1992) that sometimes also involved control of even long-distance dispersers (Inchausti and Weimerskirch 2002). This makes it unlikely that the proportion of unrecorded recruits is systematically smaller among longlived species, even though the number of local recruits may be extremely small also in long-lived species such as Pagodroma nivea (Chastel et al. 1993). In spite of these caveats, we still find that the contribution of demographic stochasticity to annual variation in population size of birds increases with decreasing life expectancy and increasing clutch size (fig. 3). This supports the hypothesis that reduced demographic stochasticity is found in species at the slow end of the slow-fast continuum of life-history variation (Sæther and Bakke 2000; Bennett and Owens 2002).

The methods employed here allow for separating out the relative contribution of demographic and environmental stochasticity to variation in annual changes in (log) population size. This represents an important extension of earlier stochastic population models that considered only either demographic stochasticity (Bartlett 1960) or environmental variance (Tuljapurkar 1990). Our results indicate a covariation across species between these two stochastic components of the population dynamics, although this relationship was strongly sensitive by one outlier (fig. 4). However, we estimated $\sigma_{\mathrm{e}}^{2}$ only for species that matured earlier than 4 years of age to avoid the effects of life-history-induced effects on the population fluctuations (Lande et al. 2002). Although we have no methods available yet for separating out this effect, our estimates suggest that a reduction of the stochastic influence on the population fluctuations may be a general characteristic of the population dynamics of long-lived species.

Analyses of avian life-history variation have identified strong phylogenetic effects (Bennett and Owens 2002). Some families have evolved slow development, delayed maturation, and small clutch sizes, whereas others are characterized by an early age of maturity and high reproductive output (Lack 1968). Accordingly, for many of the relationships, our phylogenetic analyses show high values of the phylogenetic index $\tau$ (Freckleton et al. 2002), indicating that phylogenetic relationships explain a high proportion of the relationship between demographic stochasticity and life-history variation in birds. This component was especially large for the contribution of fecundity to interspecific variation in $\sigma_{\mathrm{d}}^{2}$.

Three explanations may account for these results. First, the association between $\sigma_{\mathrm{d}}^{2}$ and the life-history characteristics (fig. 3) may be due to a relationship between habitat characteristics and population dynamics. If, for instance, marine environments are more stable than terrestrial environments, the proportion of marine species in the data set will strongly influence any relationships. However, including only terrestrial species in the analyses had only a slight effect $(<10 \%)$ on the correlation coefficients in figure 3. Second, our results (figs. 3, 4) may suggest an adaptive reduction through natural selection of the influence of stochastic variation on population variability in long-lived species. In general, the deterministic growth rate $r_{1}$ in birds decreases with adult survival rate (Sæther and Engen $2002 b$; Sæther et al. 2002a). This reduces the maximal amount of stochastic variation in the population dynamics that still ensures a positive long-run population growth rate (see eq. [2]). Accordingly, species with small clutch sizes have smaller demographic variances (fig. $3 a$ ). Since individual variation in resource allocation is likely to affect 
reproductive success (Roff 1992), this suggests that females in long-lived species allocate resources toward their own survival at the expense of expected survival of offspring under unfavorable environmental conditions. This is also supported by theoretical analyses of life-history evolution in stochastic environments showing increased environmental stochasticity to favor delayed reproduction or reduced parental effort to reduce the risk of extinction (Orzack 1997; Erikstad et al. 1998; Benton and Grant 1999; Orzack and Tuljapurkar 2000; Gaillard and Yoccoz 2003). For instance, female cervides may stop lactating during periods with bad weather (Gaillard and Yoccoz 2003), resulting in mortality of the offspring. Accordingly, shortlived bird species generally respond by increased foraging effort when the nutritional requirements are artificially increased (Wright and Cuthill 1989), but such a response sacrificing the probability of own survival in favor of offspring investment is rarely recorded in long-lived species (Sæther et al. 1993).

A general pattern that seems to emerge from several studies covering a variety of taxa (Pfister 1998; Sæther and Bakke 2000; Gaillard and Yoccoz 2003) is that smaller temporal variation is found in those demographic traits that most strongly influence variation in population growth rate. However, these studies were unable to distinguish between the relative contribution of density dependence and stochastic variation to the variance in demographic traits. Our results suggest that an important contribution in birds to such an inverse relationship between variance and sensitivity (Caswell 2001) of a trait is a reduction in long-lived species of the influences of the demographic stochasticity (fig. 3) and also maybe the environmental stochasticity (fig. 4 ) on the population growth rate. This suggests an interaction between patterns in population dynamics and evolutionary processes within populations, probably operating through an increase of life expectancy in the intensity of natural selection to reduce the influence of stochastic variation on demographic traits with high sensitivity.

\section{Acknowledgments}

We are grateful to H. van Balen, the late Huybert van Eck, and J. Visser for help with the fieldwork; the Arbeitskreis Wanderfalkenschutz (G. Kleinstäuber, Freiberg), L. Bruinzeel, A. Dhondt, B. Ullrich, M. van der Pol, S. Verhulst, and J. Visser for access to unpublished data; R. Freckleton for help with the phylogenetic analyses; and T. Coulson for comments on previous versions of the manuscript. The study was financed by grants from the European Commission (project METABIRD), the Research Council of Norway (Strategic University Program), the National Science Foundation, and the Dutch Science Foundation
(Nederlandsde Organisatie voor Wetenschappelijk Onderzoek).

\section{Literature Cited}

Ariño, A., and S. L. Pimm. 1995. On the nature of population extremes. Evolutionary Ecology 9:429-443.

Bartlett, M. S. 1960. Stochastic population models in ecology and epidemiology. Methuen, London.

Bennett, P. M., and I. P. F. Owens. 2002. Evolutionary ecology of birds. Oxford University Press, Oxford.

Benton, T. G., and A. Grant. 1999. Optimal reproductive effort in stochastic, density-dependent environments. Evolution 53:677-688.

Bro, E., F. Sarrazin, J. Clobert, and F. Reitz. 2000. Demography and the decline of the grey partridge Perdix perdix in France. Journal of Applied Ecology 37:432448.

Caswell, H. 2001. Matrix population models. 2d ed. Sinauer, Sunderland, Mass.

Chastel, O., H. Weimerskirch, and P. Jouventin. 1993. High annual variability in reproductive success and survival of an Antarctic seabird, the snow petrel Pagodroma nivea: a 27-year study. Oecologia (Berlin) 94:278-285.

Dennis, B., P. Munholland, and J. M. Scott. 1991. Estimation of growth and extinction parameters for endangered species. Ecological Monographs 61:115-143.

Engen, S., Ø. Bakke, and A. Islam. 1998. Demographic and environmental stochasticity: concepts and definitions. Biometrics 54:830-836.

Engen, S., B.-E. Sæther, and A. P. Møller. 2001. Stochastic population dynamics and time to extinction of a declining population of barn swallows. Journal of Animal Ecology 70:789-797.

Engen, S., R. Lande, and B.-E. Sæther. 2003. Demographic stochasticity and Allee effects in populations with two sexes. Ecology 84:2378-2386.

Engen, S., R. Lande, B.-E. Sæther, and T. Bregnballe. In press. Estimating the pattern of synchrony in fluctuating populations. Journal of Animal Ecology.

Erikstad, K. E., P. Fauchald, T. Tveraa, and H. Steen. 1998. On the cost of reproduction in long-lived birds: the influence of environmental variability. Ecology 79:17811788.

Fox, G. A., and B. E. Kendall. 2002. Demographic stochasticity and the variance reduction effect. Ecology 83: 1928-1934.

Freckleton, R. P., P. H. Harvey, and M. D. Pagel. 2002. Phylogenetic analysis and comparative data: a test and review of evidence. American Naturalist 160:712-726.

Gaillard, J.-M., and N. G. Yoccoz. 2003. Temporal variation in survival of mammals: a case of environmental canalization? Ecology 84:3294-3306. 
Gilpin, M. E., and F. J. Ayala. 1973. Global models of growth and competition. Proceedings of the National Academy of Sciences of the USA 70:3590-3593.

Goodman, L. 1967. The probability of extinction for birthand-death processes that are age-dependent or phasedependent. Biometrika 54:579-596.

Hansen, F. 1987. Die Einwanderung des Schwarzspechtes Dryocopus martius nach Bornholm und seine Populationsentwicklung während 25 Jahren. Acta Regiae Societatis Litteraturae Gothoburgensis, Zoologica 14:5359.

Inchausti, P., and J. Halley. 2002. The long-term temporal variability and spectral colour of animal populations. Evolutionary Ecology Research 4:1033-1048.

Inchausti, P., and H. Weimerskirch. 2002. Dispersal and metapopulation dynamics of an oceanic seabird, the wandering albatross, and its consequences for its response to long-line fisheries. Journal of Animal Ecology 71:765-770.

Lack, D. 1968. Ecological adaptations for breeding in birds. Methuen, London.

Lambrechts, M. M., J. Blondel, A. Caizergues, P. C. Dias, R. Pradel, and D. W. Thomas. 1999. Will estimates of lifetime recruitment of breeding offspring on small-scale study plots help us to quantify processes underlying adaptation? Oikos 86:147-151.

Lande, R. 1998. Demographic stochasticity and Allee effect on a scale with isotropic noise. Oikos 83:353-358.

Lande, R., S. Engen, B.-E. Sæther, F. Filli, E. Matthysen, and H. Weimerskirch. 2002. Estimating density dependence from population time series using demographic theory and life-history data. American Naturalist 159: 321-337.

Lande, R., S. Engen, and B.-E. Sæther. 2003. Stochastic population dynamics in ecology and conservation. Oxford University Press, Oxford.

Lebreton, J. 1990. Modelling density dependence, environmental variability, and demographic stochasticity from population counts: an example using Wytham Wood great tits. Pages 89-102 in J. Blondel, A. Gosler, J.-D. Lebreton, and R. H. McCleery, eds. Population biology of passerine birds. Springer, Berlin.

Leigh, E. G., Jr. 1981. The average lifetime of a population in a varying environment. Journal of Theoretical Biology 90:213-239.

May, R. M. 1973. Stability in randomly fluctuating versus deterministic environments. American Naturalist 107: 621-650.

Newton, I. 1998. Population limitation in birds. Academic Press, San Diego, Calif.

Orzack, S. H. 1997. Life history evolution and extinction. Pages 273-302 in S. Tuljapurkar and H. Caswell, eds.
Structured population models in marine, terrestrial, and freshwater systems. Chapman \& Hall, New York.

Orzack, S. H., and S. Tuljapurkar. 2000. Reproductive effort in variable environments, or environmental variation is for the birds. Ecology 82:2659-2665.

Pagel, M. D. 1999. Inferring the historical patterns of biological evolution. Nature 401:877-884.

Pfister, C. 1998. Patterns of variance in stage-structured populations: evolutionary predictions and ecological implications. Proceedings of the National Academy of Sciences of the USA 95:213-218.

Pimm, S. L. 1991. The balance of nature? University of Chicago Press, Chicago.

Roff, D. 1992. The evolution of life histories. Chapman \& Hall, New York.

Sæther, B.-E., and Ø. Bakke. 2000. Avian life history variation and contribution of demographic traits to the population growth rate. Ecology 81:642-653.

Sæther, B.-E., and S. Engen. 2002a. Including uncertainties in population viability analysis using population prediction intervals. Pages 191-212 in S. R. Beissinger and D. R. McCullough, eds. Population viability analysis. University of Chicago Press, Chicago.

- 2002b. Pattern of variation in avian population growth rates. Philosophical Transactions of the Royal Society of London B 357:1185-1196.

- 2003. The routes to extinction. Pages 218-236 in T. Blackburn and K. Gaston, eds. Macroecology. Blackwell, Oxford.

Sæther, B.-E., R. Andersen, and H. C. Pedersen. 1993. Regulation of parental effort in a long-lived seabird: an experimental manipulation of the cost of reproduction in the antarctic petrel, Thalassoica antarctica. Behavioral Ecology and Sociobiology 33:147-150.

Sæther, B.-E., S. Engen, A. Islam, R. McCleery, and C. Perrins. 1998. Environmental stochasticity and extinction risk in a population of a small songbird, the great tit. American Naturalist 151:441-450.

Sæther, B.-E., S. Engen, R. Lande, P. Arcese, and J. N. M. Smith. 2000a. Estimating the time to extinction in an island population of song sparrows. Proceedings of the Royal Society of London B 267:621-626.

Sæther, B.-E., J. Tufto, S. Engen, K. Jerstad, O. W. Røstad, and J. E. Skåtan. 2000b. Population dynamical consequences of climate change for a small temperate songbird. Science 287:854-856.

Sæther, B.-E., S. Engen, and E. Matthysen. 2002a. Demographic characteristics and population dynamical patterns of solitary birds. Science 295:2070-2073.

Sæther, B.-E., S. Engen, R. Lande, C. Both, and M. E. Visser. 2002b. Density-dependence and stochastic variation in the dynamics of a newly established small songbird population. Oikos 99:331-337. 
Sæther, B.-E., S. Engen, F. Filli, R. Aanes, W. Schröder, and R. Andersen. 2002c. Stochastic population dynamics of an introduced Swiss population of the ibex. Ecology 83:3457-3465.

Sæther, B.-E., S. Engen, A. P. Møller, E. Matthysen, F. Adriaensen, W. Fiedler, A. Leivits, et al. 2003. Climate variation and regional gradients in population dynamics of two hole nesting passerines. Proceedings of the Royal Society of London B 270:2397-2404.

Sæther, B.-E., S. Engen, R. Lande, A. P. Møller, S. Bensch, D. Hasselquist, B. Leisler, and J. Beier. 2004. Time to extinction in relation to mating system and type of density regulation in populations with two sexes. Journal of Animal Ecology 73:925-934.

Sæther, B.-E., S. Engen, A. P. Møller, M. E. Visser, E. Matthysen, W. Fiedler, M. M. Lambrechts, et al. In press. Time to extinction of bird populations. Ecology.
Sibley, C. G., and J .E. Ahlquist. 1990. Phylogeny and the classification of birds. Yale University Press, New Haven, Conn.

Stacey, P. B., and M. Taper. 1992. Environmental variation and the persistence of small populations. Ecological Application 2:18-29.

Tuljapurkar, S. D. 1990. Population dynamics in variable environments. Springer, New York.

Weimerskirch, H. 1992. Reproductive effort in long-lived birds: age-specific patterns of condition, reproduction and survival in the wandering albatross. Oikos 64:464473.

Wright, J., and I. Cuthill. 1989. Manipulation of sexdifferences in parental care. Behavioral Ecology and Sociobiology 25:171-181.

Associate Editor: Ben C. Sheldon 